\title{
Long-term outcomes of bilateral lateral rectus recession versus unilateral lateral rectus recession-medial rectus plication in children with basic type intermittent exotropia
}

\author{
Haeng-Jin Lee ${ }^{1}$ Seong-Joon $\mathrm{Kim}^{1,2} \cdot$ Young Suk Yu${ }^{1,2}$ \\ Received: 2 September 2018 / Revised: 8 February 2019 / Accepted: 8 March 2019 / Published online: 3 April 2019 \\ (c) The Royal College of Ophthalmologists 2019
}

\begin{abstract}
Purpose To compare the long-term surgical outcomes between bilateral lateral rectus recession (BLR) and unilateral lateral rectus recession-medial rectus plication (RP) in intermittent exotropia.

Methods Children who underwent BLR or RP for basic type intermittent exotropia between 2015 and 2016 with a minimum follow-up period of 2 years were retrospectively reviewed. Surgical outcomes were classified based on postoperative angle of deviation as follows: success (esodeviation $\leq 5$ prism diopters $[\mathrm{PD}]$ to exodeviation $\leq 10 \mathrm{PD}$ ), and failure (overcorrection [esodeviation $>5 \mathrm{PD}$ ] and undercorrection or recurrence [exodeviation $>10 \mathrm{PD}]$ ).

Results Of 144 patients, 90 underwent BLR and 54 underwent RP. The angle of exodeviation of the RP group steadily increased over time after the surgery. The BLR group showed an earlier exodrift and a more stable course compared to the RP group. Kaplan-Meier survival analysis showed a better survival in the BLR group, with final success rates of $48.9 \%$ in the BLR group and $25.9 \%$ in the RP group after a mean follow-up of 2.2 years. Patients with a successful outcome had greater esodeviation at 1 week postoperatively (at distance 7.6 PD in the BLR group, 11.4 in the RP group).

Conclusions Surgical outcomes were better in the BLR group than in the RP group. The RP group showed higher rates of recurrence of exodeviation, while the BLR group presented a more stable course. Establishing more esodeviation at postoperative week 1 in the RP group compared to the BLR group would be required to achieve successful results.
\end{abstract}

\section{Introduction}

Rectus muscle-strengthening procedures such as resection and plication are used to correct strabismus. Muscle resection requires muscle disinsertion and removals of part of the muscle, which is irreversible and permanently disrupts anterior ciliary circulation. Muscle plication is an alternative simple procedure that does not require cutting the muscle and therefore results in less tissue trauma. It also decreases the risk of anterior segment ischemia by preserving the anterior ciliary blood supply [1-6]. Although plication has many potential advantages over resection, a recent study

Seong-Joon Kim

ophjun@snu.ac.kr

1 Department of Ophthalmology, Seoul National University College of Medicine, Seoul, Republic of Korea

2 Seoul Artificial Eye Center, Seoul National University Hospital Clinical Research Institute, Seoul, Republic of Korea reported lower success rates and higher reoperation rates compared with resection [7]. In contrast, another study [3] reported that the effectiveness of plication was similar to that of resection and suggested plication as a good musclestrengthening procedure with vessel-sparing. However, these previous studies included various types of strabismus such as exotropia, esotropia, and hypertropia, and had varying durations of follow-up.

Intermittent exotropia is the most prevalent form of childhood exotropia [8,9]. Although the most common surgical procedures for the treatment of exotropia are bilateral lateral rectus recession (BLR) or unilateral lateral rectus recession-medial rectus resection (RR), unilateral lateral rectus recession-medial rectus plication (RP) has recently been performed due to the advantages described above. One study reported comparable success rates at 12 months for RR and RP in adult patients with intermittent exotropia [10]. However, no study has compared BLR to RP in children with intermittent exotropia. The purpose of this study was to compare the long-term surgical outcomes 
between BLR and RP in the treatment of intermittent exotropia in children.

\section{Materials and methods}

\section{Subjects}

We conducted a retrospective review of medical records for patients who underwent BLR or RP for the treatment of intermittent exotropia between January 2015 and April 2016 at Seoul National University Children's Hospital in South Korea. The minimum required follow-up period after surgery was 24 months, and patients who required reoperation within 24 months after the primary surgery were also included.

Intermittent exotropia was divided into the following types: basic, pseudo-divergence excess, true divergence excess, and convergence insufficiency. This study only included patients with basic type. The following patients were excluded from the study: patients older than 17 years of age at the time of surgery; those with a history of prior strabismus surgery; those who underwent simultaneous vertical and/or oblique muscle surgery; those with congenital or acquired ophthalmic condition (such as an optic nerve disease, glaucoma, media opacity, or cataract); and those with a systemic anomaly such as a neurologic disorder or developmental delay. Patients with dissociated vertical deviation or oblique muscle overaction that did not require surgery were included. The following patient characteristics were recorded: age at onset of deviation, age at diagnosis, age at the time of surgery, sex, birth weight, fixation preference, refractive errors, types of exotropia, associated strabismus (vertical deviation or oblique dysfunction), angle of deviation, presence of lateral incomitance, types of surgery performed (BLR or RP), stereopsis, and fusional ability. This study was approved by the Institutional Review Board of Seoul National University Hospital in South Korea and the study protocol followed the tenets of the Declaration of Helsinki.

\section{Preoperative ophthalmic examinations}

Patient age at onset of exodeviation was determined from the medical history provided by the parents. Refractive errors were determined using cycloplegia and analyzed as spherical equivalent values: myopia was represented as negative numbers and hypermetropia as positive numbers. Amblyopia was defined as a difference of at least 2 lines in visual acuity between eyes or best-corrected visual acuity lower than 20/ 30 on the Snellen visual acuity chart. Anisometropia was defined as a difference of $>1.5$ diopters (D). In patients with a fixation preference or amblyopia, part-time occlusion therapy was prescribed with spectacles if needed.
The angle of deviation was measured by alternate prism cover test with accommodative targets for fixation both at near $(0.33 \mathrm{~m})$ and at distance $(6 \mathrm{~m})$ and indicated as prism diopters (PD) with exodeviation represented as positive numbers and esodeviation as negative numbers. At distance, the angle of deviation was measured at the primary position and both right and left lateral gaze. A base in prism was placed over the adducting eye in measurements of lateral gaze deviations. Lateral incomitance was defined as more than $20 \%$ decrease in right or left gaze from the primary position.

Patients were classified as having a basic intermittent exotropia if the distance deviation was within 10 PD of the near. Divergence excess intermittent exotropia was defined as a distance deviation of at least 10 PD greater than near. Pseudo-divergence excess was defined as an exotropia that met criteria for divergence excess, but had an increase in the near deviation to an amount within $10 \mathrm{PD}$ of the distance deviation after 1-hour monocular occlusion or with the use of +3.0 diopter lenses. Convergence insufficiency was defined as an intermittent exotropia at near measuring at least 10 PD greater than that at distance. Preoperative measurements of the angle of deviation were performed on at least 3 different occasions by 1 experienced examiner (S-JK). The presence of fixation preference was determined by repeated examinations of the cover-uncover test.

Stereoacuity was tested with Titmus stereotest (Stereo Optical, Chicago, Illinois, USA) and all values were transformed to $\log$ arcsec for the purpose of analysis. Fusional ability was measured using the Worth Four-Dot Test at distance and near where cooperation was adequate.

\section{Surgical procedures}

All the surgeries were performed under general anesthesia by the single surgeon (S-JK). The selection of surgical procedure was made by the surgeon with a tendency of BLR in patients with large angle ( $240 \mathrm{PD}$ ) exotropia to avoid severe lateral incomitance, and RP in patients with fixation preference. In case of smaller angle exotropia without fixation preference, BLR or RP was performed after discussing unilateral versus bilateral surgery with the patients and their parents. Based on the maximal preoperative angle of deviation during follow-up, BLR or RP was performed in all patients using a surgical formula based on the surgeon's experience (Table 1). In case of medial rectus plication, the muscle was isolated on a hook and Tenon's tissue around the muscle insertion was removed from the sclera after performing a fornix conjunctival incision. The desired amount of plication was measured from the muscle insertion using calipers. Locking bites were made using 2 single-armed 6-0 polyglactin 910 sutures passed though both edges of the muscle at a distance from 
Table 1 Surgical table of bilateral lateral rectus recession and unilateral lateral rectus recession-medial rectus plication in children with basic type intermittent exotropia

\begin{tabular}{llll}
\hline Deviation & BLR recession $(\mathrm{mm})$ & $\begin{array}{l}\text { LR Recession / } \\
\text { MR Plication } \\
(\mathrm{mm})\end{array}$ \\
\hline 20 & 5.5 & 5.5 & 5.5 \\
25 & 6 & 6.0 & 6.0 \\
30 & 6.5 & 6.5 & 6.5 \\
35 & 7.5 & 7.5 & 7.0 \\
40 & 8.5 & 8.0 & 7.5 \\
45 & 9 & & \\
50 & 9.5 & & \\
\hline
\end{tabular}

$\overline{B L R}$ bilateral lateral rectus recession, $L R$ lateral rectus muscle, $M R$ medial rectus muscle

the insertion corresponding to the selected amount of plication. These sutures were then attached to sclera just anterior to the muscle insertion (muscle-to-sclera) by passing a partial-thickness of the sclera, and not through muscle or tendon. These sutures were tied over an iris spatula that folded the anterior part of muscle posteriorly and flattened it between the globe and the more posterior part of muscle that was sutured to the sclera. Lastly, the incised conjunctiva was arranged and wrapped around the sclera at the original position and sutured with $8-0$ polyglactin 910. In patient who showed fixation preference, the surgery was performed on the non-dominant eye. Otherwise, the surgery was performed on one randomly selected eye when no fixation preference was observed.

\section{Postoperative measurements}

Postoperative alignment at distance and near fixation in the primary position was measured at week 1, 6, 12, and 24 months and afterwards. Patients with diplopia associated with postoperative esotropia were managed by alternating full-time patching for 1 to 4 weeks until diplopia was resolved. If the esotropia did not show a reduction in 4 weeks, base-out prism glasses were prescribed to allow constant fusion until the esotropia was resolved.

\section{Assessment of surgical outcomes}

Surgical outcomes were classified into 2 groups based on postoperative angle of deviation: success (esodeviation $\leq 5$ prism diopters $[\mathrm{PD}]$ to exodeviation $\leq 10 \mathrm{PD}$ ) and failure (overcorrection [esodeviation $>5 \mathrm{PD}$ ], and undercorrection or recurrence [exodeviation $>10 \mathrm{PD}$ ]). Reoperation for recurrent exotropia was performed when the maximal angle of deviation was more than $15 \mathrm{PD}$ of exotropia with poor fusional control so that increased manifest phase of exotropia was more frequently noticed by patients, parents, or clinicians. Another indication for reoperation was consecutive constant esotropia of more than 20 PD persisting for more than 6 months, but this did not occur in this study.

\section{Statistical analysis}

Statistical analyses were performed using SPSS software (version 23 for Windows; SPSS, Chicago, IL, USA). The independent Student's $t$ test, $X^{2}$ test, and Fisher's exact test were used to compare the patient's characteristics and the surgical outcomes. A logistic regression analysis was used to investigate factors associated with recurrence. KaplanMeier survival analysis and log-rank test were used for comparison of the recurrence rate. $P$ values $<0.05$ were considered statistically significant.

\section{Results}

A total of 144 patients were included in this study (BLR, 90; RP, 54). Table 2 details the demographic and clinical characteristics of the two groups.

\section{Postoperative deviations during follow-up period}

At postoperative week 1 , deviation at distance was $-4.9 \pm$ 6.3 PD in the BLR group and $-7.0 \pm 6.1 \mathrm{PD}$ in the RP group $(p=0.050)$. Thereafter, deviations at distance became more exotropic in the RP group than in the BLR group, with a statistical significance at 1 and 2 years following the procedure ( $p=0.001$, and 0.001 , respectively). Deviation at near at postoperative week 1 was $-4.9 \pm 6.9$ PD in the BLR group and $-6.1 \pm 6.6$ PD in the RP group ( $p$ $=0.312$ ). Deviations at near became more exotropic in the RP group with a statistical significance at 6 months; and 1 and 2 years $(p=0.002,<0.001$, and $<0.001$, respectively). On the last examination, final angle of deviation at distance and near became more exotropic in the RP group than in the BLR group ( $p=0.001$, and $<0.001$, respectively). Differences between distance and near at final follow-up were $-1.6 \pm 4.8 \mathrm{PD}$ in the BLR group and $-3.5 \pm 5.8 \mathrm{PD}$ in the RP group ( $p=0.034$; Table 3$)$.

Patients in the BLR group showed an exodrift in the early phase and a more stable course with less exodrift during follow-up compared to those in the RP group. In contrast, the angle of exodeviation in the RP group steadily increased over time after the surgery (Fig. 1a).

\section{Postoperative recurrence rate and final surgical success}

The proportion of patients with overcorrection gradually decreased while that of recurrence increased in both groups 
Table 2 Comparison of demographics and clinical characteristics in the bilateral lateral rectus recession group and the unilateral lateral rectus recession-medial rectus plication group in children with basic type intermittent exotropia

\begin{tabular}{|c|c|c|c|}
\hline Variables & $\operatorname{BLR}(n=90)$ & $\mathrm{RP}(n=54)$ & $P$ value \\
\hline Age at onset of deviation (years) & $2.2 \pm 1.7(0.3-9)$ & $2.7 \pm 1.7(0.5-9.5)$ & $0.088^{\mathrm{a}}$ \\
\hline Sex (male:female) & 45: 45 & 27: 27 & $0.568^{\mathrm{b}}$ \\
\hline Gestational age (months) & $38.5 \pm 2.6(28-42)$ & $39.1 \pm 2.2(27-42)$ & $0.142^{\mathrm{a}}$ \\
\hline Weight at birth $(\mathrm{kg})$ & $3.1 \pm 0.6(1.3-4.2)$ & $3.2 \pm 0.5(0.9-4.2)$ & $0.511^{\mathrm{a}}$ \\
\hline \multicolumn{4}{|l|}{ Cycloplegic refractive errors (diopters) } \\
\hline Right eye & $0.11 \pm 1.43(-5.38-6.25)$ & $0.06 \pm 1.38(-5.25-6)$ & $0.830^{\mathrm{a}}$ \\
\hline Left eye & $0.30 \pm 0.37(0-2.5)$ & $0.41 \pm 0.75(0-5)$ & $0.259^{\mathrm{a}}$ \\
\hline Anisometropia $(n, \%)$ & $2(2.2 \%)$ & $2(3.7 \%)$ & $0.482^{\mathrm{c}}$ \\
\hline Amblyopia $(n, \%)$ & $1(1.1 \%)$ & $3(5.6 \%)$ & $0.148^{\mathrm{c}}$ \\
\hline Fixation preference $(n, \%)$ & $59(65.6 \%)$ & $52(96.3 \%)$ & $<0.001^{\text {b }}$ \\
\hline \multicolumn{4}{|l|}{ Preoperative angle of deviation (PD) } \\
\hline Maximum at distance & $31.6 \pm 6.0(20-50)$ & $28.0 \pm 5.0(20-42.5)$ & $<0.001^{\text {a }}$ \\
\hline Maximum at near & $32.4 \pm 6.1(20-50)$ & $32.1 \pm 4.4(25-40)$ & $0.720^{\mathrm{a}}$ \\
\hline Distance - near & $-0.8 \pm 3.1(-7.5-5.0)$ & $-4.2 \pm 3.3(-7.5-7.5)$ & $<0.001^{\mathrm{a}}$ \\
\hline \multicolumn{4}{|l|}{ Associated features } \\
\hline DVD & $1(1.1 \%)$ & $1(1.9 \%)$ & $0.611^{\mathrm{c}}$ \\
\hline IO overaction & $3(3.3 \%)$ & $2(3.7 \%)$ & $0.622^{\mathrm{c}}$ \\
\hline Lateral incomitancy $(n, \%)$ & $24 / 87(27.6 \%)$ & $14 / 52(26.9 \%)$ & $0.547^{\mathrm{b}}$ \\
\hline Stereopsis (logarcsec) & $1.8 \pm 0.3(1.6-2.6)$ & $1.9 \pm 0.3(1.6-2.6)$ & $0.734^{\mathrm{a}}$ \\
\hline \multicolumn{4}{|l|}{ Fusional ability } \\
\hline Distance (fusion:suppresion:diplopia) & $27: 19: 2$ & $16: 15: 4$ & $0.276^{\mathrm{b}}$ \\
\hline Near (fusion:suppresion:diplopia) & $37: 8: 3$ & 23:5:7 & $0.134^{\mathrm{b}}$ \\
\hline Age at surgery (years) & $6.1 \pm 1.8(3.1-12.2)$ & $6.6 \pm 2(3.3-14.7)$ & $0.100^{\mathrm{a}}$ \\
\hline Duration between onset and surgery (years) & $3.9 \pm 1.5(1.8-11.6)$ & $3.9 \pm 1.2(0.5-7)$ & $0.944^{\mathrm{a}}$ \\
\hline Follow-up period after surgery (months) & $26.1 \pm 3.2(24-37.4)$ & $26.2 \pm 3.4(24-36.3)$ & $0.804^{\mathrm{a}}$ \\
\hline
\end{tabular}

Values are presented as the mean \pm standard deviation (range) or number (\%)

Boldface values denote statistically significant results

$B L R$ bilateral lateral rectus recession, $R P$ unilateral lateral rectus recession-medial rectus plication, $P D$ prism diopters, $D V D$ dissociated vertical deviation, $I O$ inferior oblique

${ }^{a}$ Independent $t$ test

${ }^{b} X^{2}$ test

'Fisher's exact test

Table 3 Postoperative angle of deviation in the bilateral lateral rectus recession group and the unilateral lateral rectus recession-medial rectus plication group in patients with basic type intermittent exotropia

\begin{tabular}{|c|c|c|c|c|c|c|c|c|c|}
\hline \multirow[b]{2}{*}{ Time after surgery } & \multicolumn{3}{|c|}{ Deviation at distance (PD) } & \multicolumn{3}{|c|}{ Deviation at near (PD) } & \multicolumn{3}{|c|}{$\begin{array}{l}\text { Differences between distance and near } \\
\text { (PD) }\end{array}$} \\
\hline & BLR & $\mathrm{RP}$ & $P$ value & BLR & $\mathrm{RP}$ & $P$ value & BLR & $\mathrm{RP}$ & $P$ value \\
\hline 1 week & $-4.9 \pm 6.3$ & $-7.0 \pm 6.1$ & 0.050 & $-4.9 \pm 6.9$ & $-6.1 \pm 6.6$ & 0.312 & $0.1 \pm 3.3$ & $-0.9 \pm 3.1$ & 0.106 \\
\hline 1 month & $3.0 \pm 5.0$ & $2.4 \pm 4.4$ & 0.431 & $3.5 \pm 5.6$ & $4.0 \pm 6.1$ & 0.585 & $-0.5 \pm 3.0$ & $-1.7 \pm 3.7$ & 0.035 \\
\hline 6 months & $4.6 \pm 7.4$ & $6.8 \pm 6.1$ & 0.076 & $5.5 \pm 8.1$ & $9.7 \pm 7.5$ & 0.002 & $-0.8 \pm 3.0$ & $-2.9 \pm 3.3$ & $<0.001$ \\
\hline 1 year & $6.9 \pm 8.2$ & $11.6 \pm 7.5$ & 0.001 & $7.6 \pm 9.1$ & $14.3 \pm 8.5$ & $<0.001$ & $-0.7 \pm 3.4$ & $-2.7 \pm 3.3$ & 0.001 \\
\hline 2 years & $9.8 \pm 9.0$ & $14.7 \pm 8.1$ & 0.001 & $11.2 \pm 11.0$ & $18.1 \pm 10.4$ & $<0.001$ & $-1.5 \pm 4.9$ & $-3.4 \pm 5.0$ & 0.022 \\
\hline Final & $10.1 \pm 9.1$ & $15.4 \pm 8.3$ & 0.001 & $11.7 \pm 11.2$ & $18.9 \pm 10.8$ & $<0.001$ & $-1.6 \pm 4.8$ & $-3.5 \pm 5.8$ & 0.034 \\
\hline
\end{tabular}

All $P$ values were by independent $t$ test

Values are presented as the mean \pm standard deviation

Boldface values denote statistically significant results

$P D$ prism diopters, $B L R$ bilateral lateral rectus recession, $R P$ unilateral lateral rectus recession-medial rectus plication 

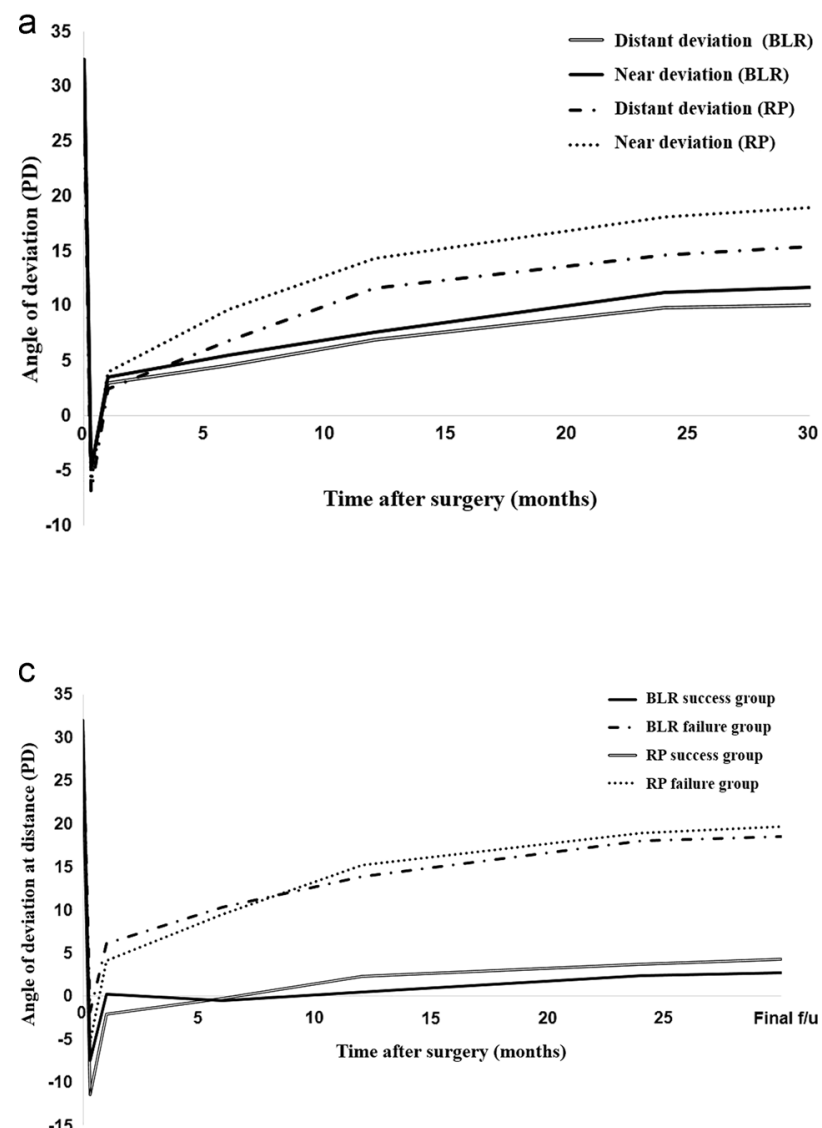

Fig. 1 a Courses of angle of deviation in patients with intermittent exotropia. b Kaplan-Meier survival analysis for recurrence of exodeviation more than 10PD. c Courses of angle of distant deviation in patients with intermittent exotropia by dividing the surgical success at

following the surgery. At week 1 postoperatively, the rate of overcorrection at distance was $42.2 \%$ in the BLR group and $61.1 \%$ in the RP group; and at near, $43.3 \%$ in the BLR group and $51.9 \%$ in the RP group. However, the recurrence rate progressively increased during follow-up in the RP group and the final surgical success at distance was $48.9 \%$ in the BLR group and $25.9 \%$ in the RP group $(p=0.021$; Table 4).

Kaplan-Meier survival analysis for recurrence of exodeviation more than 10 PD showed a significant difference in the cumulative probability of surgical success, with better survival in the BLR group $(p=0.012$, log-rank test; Fig. 1b). Out of all the patients in this study, 10 underwent reoperation for recurrence (BLR, 3; RP, 7).

\section{Factors associated with surgical outcomes}

There was no significant difference in preoperative clinical factors including sex, age, refractive errors, and stereopsis between the 2 groups. These clinical factors were also not significantly associated with recurrence on logistic regression analysis. However, the amount of initial overcorrection
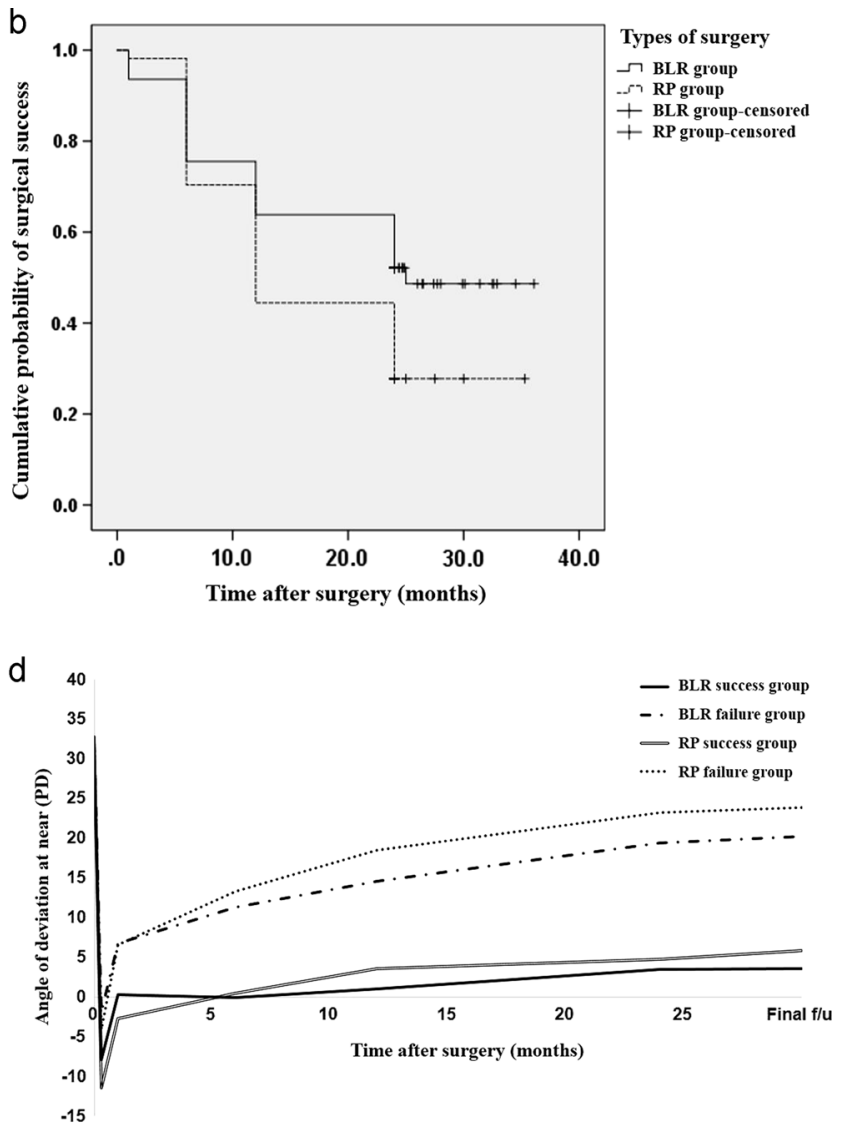

postoperative 2 year. $\mathbf{d}$ Courses of angle of near deviation in patients with intermittent exotropia by dividing the surgical success at postoperative 2 year

was associated with final success $(p=0.027)$. Patients with a successful outcome at 2 years had greater esodeviation at postoperative week 1 than those with failure outcome. The mean distant esodeviation at postoperative week 1 was 7.6 $\pm 6.5 \mathrm{PD}$ in the success group and 2.0 $\pm 4.5 \mathrm{PD}$ in the failure group for patients who underwent BLR; and $11.4 \pm 6.2 \mathrm{PD}$ and 5.3 \pm 5.2 PD respectively for those who underwent RP (Fig. 1c). Mean near esodeviation at postoperative week 1 was 7.9 $\pm 7.6 \mathrm{PD}$ in the success group and $1.8 \pm 4.3 \mathrm{PD}$ in the failure group for patients who underwent BLR group; and $11.4 \pm 6.8 \mathrm{PD}$ and $4.1 \pm 5.3 \mathrm{PD}$ respectively for patients who underwent RP (Fig. 1d).

\section{Discussion}

This study compared long-term surgical outcomes in patients with intermittent exotropia treated with BLR and RP. The RP group showed a higher rate of overcorrection in the early postoperative period, but recurrence rates gradually increased over time. In contrast, the BLR group showed an earlier exodrift followed by a stable course during 
Table 4 Postoperative surgical outcomes in the bilateral lateral rectus recession group and the unilateral lateral rectus recession-medial rectus plication group in patients with basic type intermittent exotropia

\begin{tabular}{|c|c|c|c|c|c|c|c|}
\hline \multirow[b]{2}{*}{$\begin{array}{l}\text { Time after } \\
\text { surgery }\end{array}$} & \multirow[b]{2}{*}{ Surgical outcome } & \multicolumn{3}{|l|}{ Distance } & \multicolumn{3}{|l|}{ Near } \\
\hline & & BLR & $\mathrm{RP}$ & $P$ value & BLR & $\mathrm{RP}$ & $P$ value \\
\hline \multirow[t]{3}{*}{1 week } & Success & $52(57.8 \%)$ & $21(38.9 \%)$ & 0.039 & $51(55.4 \%)$ & $26(48.1 \%)$ & 0.389 \\
\hline & Undercorrection & $0(0 \%)$ & $0(0 \%)$ & & $0(0 \%)$ & $0(0 \%)$ & \\
\hline & Overcorrection & $38(42.2 \%)$ & $33(61.1 \%)$ & & $39(43.3 \%)$ & $28(51.9 \%)$ & \\
\hline \multirow[t]{3}{*}{1 month } & Success & $79(87.8 \%)$ & $50(92.6 \%)$ & 0.531 & $77(85.6 \%)$ & $43(79.6 \%)$ & 0.613 \\
\hline & Recurrence & $5(5.6 \%)$ & $1(1.9 \%)$ & & $8(8.9 \%)$ & $6(11.1 \%)$ & \\
\hline & Overcorrection & $6(6.7 \%)$ & $3(5.6 \%)$ & & $5(5.6 \%)$ & $5(9.3 \%)$ & \\
\hline \multirow[t]{3}{*}{6 months } & Success & $63(70.0 \%)$ & $36(66.7 \%)$ & 0.727 & $62(68.9 \%)$ & $26(48.1 \%)$ & 0.014 \\
\hline & Recurrence & $22(24.4 \%)$ & $16(29.6 \%)$ & & $22(24.4 \%)$ & $26(48.1 \%)$ & \\
\hline & Overcorrection & $5(5.6 \%)$ & $2(3.7 \%)$ & & $6(6.7 \%)$ & $2(3.7 \%)$ & \\
\hline \multirow[t]{3}{*}{1 year } & Success & $54(60.0 \%)$ & $23(42.6 \%)$ & 0.058 & $57(63.3 \%)$ & $16(29.6 \%)$ & $<0.001$ \\
\hline & Recurrence & $32(35.6 \%)$ & $30(55.6 \%)$ & & $30(33.3 \%)$ & $38(70.4 \%)$ & \\
\hline & Overcorrection & $4(4.3 \%)$ & $1(1.9 \%)$ & & $3(3.3 \%)$ & $0(0 \%)$ & \\
\hline \multirow[t]{3}{*}{2 years } & Success & $45(50.0 \%)$ & $14(25.9 \%)$ & 0.015 & $45(50.0 \%)$ & $13(24.1 \%)$ & 0.008 \\
\hline & Recurrence & $43(47.8 \%)$ & $39(72.2 \%)$ & & $43(47.8 \%)$ & $40(74.1 \%)$ & \\
\hline & Overcorrection & $2(2.2 \%)$ & $1(1.9 \%)$ & & $2(2.2 \%)$ & $1(1.9 \%)$ & \\
\hline \multirow[t]{3}{*}{ Final } & Success & $44(48.9 \%)$ & $14(25.9 \%)$ & 0.022 & $44(48.9 \%)$ & $12(22.2 \%)$ & 0.006 \\
\hline & Recurrence & $44(48.9 \%)$ & $39(72.2 \%)$ & & $44(48.9 \%)$ & $41(75.9 \%)$ & \\
\hline & Overcorrection & $2(2.2 \%)$ & $1(1.9 \%)$ & & $2(2.2 \%)$ & $1(1.9 \%)$ & \\
\hline
\end{tabular}

All $P$ values were by Fisher's exact test

Values are presented as number $(\%)$

Boldface values denote statistically significant results

$B L R$ bilateral lateral rectus recession, $R P$ unilateral lateral rectus recession-medial rectus plication follow-up. After a mean follow-up period of 2.2 years, the BLR group showed a higher success rate compared with the RP group. There have been many reported comparisons between BLR and RR [11-15]. We also previously conducted a comparison between the BLR and RR and found that BLR presented better long-term surgical outcomes compared with RR [11]. Previous studies, including our study, have reported that RR presents a greater degree of subsequent exodrift than does BLR [11, 16, 17]. Likewise, continuous exodrift occurred similarly in the RP group in the present study.

Several strabismus experts have recently adopted muscle plication based on many potential benefits over resection. Chaudhuri and Demer [3] found similar effectiveness in exotropia correction with resection or plication of the medial rectus muscle, but $37 \%$ less effectiveness with plication of the lateral rectus muscle on long-term follow-up compared to resection. In contrast, Alkharashi and Hunter [7] reported lower intermediate- and long-term success rates with rectus muscle plication compared to resection. We have been using plication in our practice for the past 6 years. Initially, we noted more frequent reoperations among patients who underwent plication compared to resection, and less effectiveness of plication compared to an equivalent amount of muscle resection. Therefore, we augmented the surgical dosage by $1.0-1.5 \mathrm{~mm}$ over the original surgical formula of resection. We used this approach in the current study and found that both the angle of esodeviation at 1 postoperative week and overcorrection were larger in the RP group compared to the BLR group. Although we augmented the surgical dosage of RP, which led to a higher and sufficient overcorrection rate in the early phase, exodrift progressively occurred over time in the RP group. Even if this needs careful consideration, augmenting the surgical dosage further in the plication might be required in order to increase success rates at final follow-up. In addition, as larger overcorrected esodeviation angles could lead to persistent postoperative diplopia, which disrupts fusion in children, it could be prevented by wearing appropriately prescribed prism glasses after surgery.

As plication is one of the rectus muscle-strengthening procedures, an initial tethering effect was observed, which led to the success in the early phase. However, the tethering effect decreased over time and the persistent tension on medial rectus resulted in a subsequent exodrift similar to that seen with resection [11]. Another possible explanation of changes in angles over time is muscle length adaptation by Guyton and colleages [18-20]. In response to the sustained divergence stimulation, shortened medial rectus gradually went through the muscle lengthening, resulting in 
increasing exotropia over time. This also demonstrates that mechanisms of action and postoperative change of deviation angle after muscle-strengthening surgery are different from those occurring after muscle-weakening surgery such as recession.

Although the initial tethering effect causes early overcorrection after resection or plication, Kimura et al. [10]. compared two procedures in adult patients and speculated that the tethering effect could be more weakened after resection due to inflammation and adhesion. They asserted that the less invasive plication procedure might lead to better results in the early postoperative period. Wright [21] also emphasized that resection is muscle-strengthening while plication is a distinct muscle-tightening procedure. However, Huston et al. [2]. found that horizontal rectus muscle plication produced similar changes in ocular alignment and surgical success compared to rectus muscle resection at 4-16 weeks after surgery when coupled with comparable amounts of antagonist muscle recession. Therefore, further comparison of surgical outcomes between plication and resection in children with intermittent exotropia is necessary.

In the present study, preoperative maximal angle of deviation at distance was larger in the BLR group than in the RP group. This difference in preoperative angle and surgical amount might have affected the surgical outcome [22]. However, immediate postoperative angle of deviation at distance was similar in both groups, and there was no association between preoperative maximal angle of deviation and final surgical success. As with other studies that have not found a significant influence of the preoperative amount of exodeviation on the surgical outcome [16, 23], this preoperative difference also had minimal impact on the surgical outcome in this study.

Initial postoperative deviation is considered an associated factor for surgical success in intermittent exotropia, and many studies have reported that initial postoperative overcorrection provides long-term stability of ocular alignment $[24,25]$. However, the amount of immediate overcorrection is still controversial. Scott et al. [26]. reported that slight overcorrection of 4-14 PD at immediate postoperative measurement yields the best result; while Richard and Parks [27] recommended 10-15 PD; Knapp et al. [28]. recommended 11-20 PD. In the present study, patients with a successful outcome presented with greater esodeviation at postoperative week 1 and showed a more stable course with less exodrift than those with surgical failure. This finding suggests that early postoperative overcorrection is beneficial for the maintenance of successful surgical outcome in patients with intermittent exotropia. In addition, the distant esodeviation at 1 week among patients with a successful outcome was larger in the RP group than in the BLR group (BLR, 7.6 PD; RP, 11.4 PD); better surgical outcomes following RP could be anticipated if alignment of $>10$ PD esodeviation is achieved at postoperative week 1. Because patients in the RP group also presented more exodrift than BLR, establishing more esodeviation at postoperative week 1 in the RP group compared to the BLR group is important to achieve successful results.

Although we only included the patients with basic type of intermittent exotropia, distance-near preoperative angle disparity was larger in the RP group. As this was a retrospective study, patients were not randomly assigned to each procedure. This resulted in a tendency to choose the RP procedure for patients with larger deviation at near. Burian and Spivey [29] recommended RR as the preferred surgical procedure for the basic type based on the theory that the RR procedure would affect the distance and near deviation equally, whereas BLR would affect the distance deviation more than the near deviation. In this study, deviation at near became larger than deviation at distance in both groups after surgery, especially in the RP group. However, distance-near differences did not significantly increase in this study, and even slightly decreased after surgery. We found that RP could maintain or reduce the distance-near disparity effectively in the basic type of intermittent exotropia.

The final recurrence rates were relatively high at $48.9 \%$ in the BLR group and $72.2 \%$ in the RP group after a mean of 2.2 years of follow-up. Usually, the success after strabismus surgery is determined by deviation angle and fusional control. However, the surgical outcome in this study was assessed only as motor outcome measured by alternate prism cover test. Therefore, patients who had exophoria of more than 10 PD were classified as recurrence, despite having good fusional control. The recurrence rate does not always indicate surgical failure or unsatisfactory results and should be distinguished from the reoperation rate. In this study, only 9 patients of 83 patients with recurrence at final follow-up had reoperation. In addition, the relatively long follow-up duration of more than 2 years in our study may have contributed to the higher reported recurrence rates.

Basic type intermittent exotropia is the subtype for which there is the most disagreement regarding the optimal surgical approach. Several studies demonstrated the superiority of BLR surgery over RR surgery $[11,16,17]$. It has been pointed out more subsequent exodrift following RR. However, RR or RP are still preferable, or should we choose the RR or RP when exotropia recurs after BLR or contralateral $\mathrm{RR}$. This is the first study to compare long-term surgical outcomes between BLR and RP in children with basic type intermittent exotropia. We analyzed the angle, not only at distance, but also at near and evaluated the distance-near disparity. Establishing patterns of postoperative changes in deviation angle and defining optimal esodeviation would be helpful in the treatment of patients with intermittent exotropia. 
There are some limitations in this study. First, this was a retrospective study and patients with younger age, poor cooperation, or lack of documentation were therefore excluded. There would be a selection bias in the choice of surgical procedure. However, patients in both groups were recruited over the same period. Second, despite setting the minimum follow-up as 2 years, there were variations in the final follow-up period between patients. Of particular note was a tendency for patients with successful results to stop visiting the clinic, while patients with unfavorable results were followed-up for a longer duration, resulting in higher reporting of recurrence rates. A prospective study with long-term follow-up exceeding 2 years would address this challenge.

In conclusion, surgical outcomes at final follow-up were better for patients with intermittent exotropia treated with BLR compared to those treated with RP. Persistent exodrift leading to higher recurrence occurred in the RP group, while the BLR group presented with earlier exodrift followed by a stable course. To achieve favorable surgical outcomes for intermittent exotropia, RP needs more overcorrection at postoperative week 1 than BLR.

\section{Summary}

\section{What was known before}

- Intermittent exotropia is one of the most prevalent forms of childhood exotropia. There have been previous studies investigating the surgical outcomes and associated factors in patients with intermittent exotropia after performing bilateral lateral rectus recession (BLR) or unilateral lateral rectus recession-medial rectus resection, which are the most common surgical procedures for the treatment of exotropia. Muscle plication does not require cutting the muscle leading to less tissue trauma. Recently, due to advantages such as sparing anterior ciliary circulation, unilateral lateral rectus recessionmedial rectus plication (RP) has been performed instead of resection. However, no study has compared the surgical outcomes of BLR to RP in children with basic type intermittent exotropia.

\section{What this study adds}

- We evaluated the long-term surgical outcomes between BLR and RP in the treatment of basic type intermittent exotropia in children. Surgical outcomes at final followup were better for patients with BLR. compared to those treated with RP. The recurrence rates of RP group gradually increased over time. In contrast, the BLR group showed an earlier exodrift followed by a stable course. To achieve favorable surgical outcomes for intermittent exotropia, RP needs more overcorrection at postoperative week 1 than the BLR. Since several strabismus experts have adopted the muscle plication, these findings would be useful in performing the surgical treatment in children with intermittent exotropia.

\section{Compliance with ethical standards}

Conflict of interest The authors declare that they have no conflict of interest.

Publisher's note: Springer Nature remains neutral with regard to jurisdictional claims in published maps and institutional affiliations.

\section{References}

1. Wright $\mathrm{KW}$, Lanier $\mathrm{AB}$. Effect of a modified rectus tuck on anterior segment circulation in monkeys. J Pediatr Ophthalmol Strabismus. 1991;28:77-81.

2. Huston PA, Hoover DL. Surgical outcomes following rectus muscle plication versus resection combined with antagonist muscle recession for basic horizontal strabismus. J AAPOS. 2018;22:7-11. https://doi.org/10.1016/j.jaapos.2017.09.004

3. Chaudhuri Z, Demer JL. Surgical outcomes following rectus muscle plication: a potentially reversible, vessel-sparing alternative to resection. JAMA Ophthalmol. 2014;132:579-85. https:// doi.org/10.1001/jamaophthalmol.2013.8188

4. Oltra EZ, Pineles SL, Demer JL, Quan AV, Velez FG. The effect of rectus muscle recession, resection and plication on anterior segment circulation in humans. $\mathrm{Br} \mathrm{J}$ Ophthalmol. 2015;99:556-60. https://doi.org/10.1136/bjophthalmol-2014305712

5. Mojon DS. Comparison of a new, minimally invasive strabismus surgery technique with the usual limbal approach for rectus muscle recession and plication. Br J Ophthalmol. 2007;91:76-82. https://doi.org/10.1136/bjo.2006.105353

6. Velez FG, Demer JL, Pihlblad MS, Pineles SL. Rectus muscle plication using an adjustable suture technique. J AAPOS. 2013;17:480-3. https://doi.org/10.1016/j.jaapos.2013.06.002

7. Alkharashi M, Hunter DG. Reduced surgical success rate of rectus muscle plication compared to resection. J AAPOS. 2017;21:2014. https://doi.org/10.1016/j.jaapos.2017.05.012

8. Mohney BG, Huffaker RK. Common forms of childhood exotropia. Ophthalmology. 2003;110:2093-6. https://doi.org/10. 1016/j.ophtha.2003.04.001

9. Govindan M, Mohney BG, Diehl NN, Burke JP. Incidence and types of childhood exotropia: a population-based study. Ophthalmology. 2005;112:104-8. https://doi.org/10.1016/j.ophtha. 2004.07.033

10. Kimura Y, Kimura T. Comparative study of plication-recession versus resection-recession in unilateral surgery for intermittent exotropia. Jpn J Ophthalmol. 2017;61:286-91. https://doi.org/10. 1007/s10384-017-0501-5

11. Choi J, Chang JW, Kim SJ, Yu YS. The long-term survival analysis of bilateral lateral rectus recession versus unilateral recession-resection for intermittent exotropia. Am J Ophthalmol. 2012;153:343-.e341. https://doi.org/10.1016/j.ajo.2011.06.024

12. Jeoung JW, Lee MJ, Hwang JM. Bilateral lateral rectus recession versus unilateral recess-resect procedure for exotropia with a 
dominant eye. Am J Ophthalmol. 2006;141:683-8. https://doi.org/ 10.1016/j.ajo.2005.11.021

13. Suh SY, Choi J, Kim SJ. Comparative study of lateral rectus recession versus recession-resection in unilateral surgery for intermittent exotropia. J AAPOS. 2015;19:507-11. https://doi.org/ 10.1016/j.jaapos.2015.08.011

14. Wang L, Wu Q, Kong X, Li Z. Comparison of bilateral lateral rectus recession and unilateral recession resection for basic type intermittent exotropia in children. Br J Ophthalmol. 2013;97:870 3. https://doi.org/10.1136/bjophthalmol-2013-303167

15. Kim KE, Yang HK, Hwang JM. Comparison of long-term surgical outcomes of 2-muscle surgery in children with large-angle exotropia: bilateral vs unilateral. Am J Ophthalmol. 2014;157:1214-.e1212. https://doi.org/10.1016/j.ajo.2014.02.038

16. Chia A, Seenyen L, Long QB. Surgical experiences with twomuscle surgery for the treatment of intermittent exotropia. J AAPOS. 2006;10:206-11. https://doi.org/10.1016/j.jaapos.2005. 11.015

17. Maruo T, Kubota N, Sakaue T, Usui C. Intermittent exotropia surgery in children: long term outcome regarding changes in binocular alignment. A study of 666 cases. Binocul Vis Strabismus Q. 2001;16:265-70.

18. Wright WW, Gotzler KC, Guyton DL. Esotropia associated with early presbyopia caused by inappropriate muscle length adaptation. J AAPOS. 2005;9:563-6. https://doi.org/10.1016/j.jaapos. 2005.06.008

19. Guyton DL. The 10th Bielschowsky Lecture. Changes in strabismus over time: the roles of vergence tonus and muscle length adaptation. Binocul Vis Strabismus Q. 2006;21:81-92.
20. Guyton DL. Ocular torsion reveals the mechanisms of cyclovertical strabismus: the Weisenfeld lecture. Invest Ophthalmol Vis Sci. 2008;49:847-57, 846. https://doi.org/10.1167/iovs.070739

21. Wright KW. Rectus muscle plication procedure. JAMA Ophthalmol. 2015;133:226-7. https://doi.org/10.1001/jamaophtha lmol.2014.4259

22. Kushner BJ, Fisher MR, Lucchese NJ, Morton GV. Factors influencing response to strabismus surgery. Arch Ophthalmol. 1993;111:75-9.

23. Koklanis K, Georgievski Z. Recurrence of intermittent exotropia: factors associated with surgical outcomes. Strabismus. 2009;17:37-40. https://doi.org/10.1080/09273970802678750

24. Oh JY, Hwang JM. Survival analysis of 365 patients with exotropia after surgery. Eye. 2006;20:1268-72. https://doi.org/10. 1038/sj.eye.6702091

25. Jampolsky A. Surgical correction of overcorrections in strabismus. Am Orthopt J. 1976;26:14-9.

26. Scott WE, Keech R, Mash AJ. The postoperative results and stability of exodeviations. Arch Ophthalmol. 1981;99:1814-8.

27. Richard JM, Parks MM. Intermittent exotropia. Surgical results in different age groups. Ophthalmology. 1983;90:1172-7.

28. Knapp P (1971) Management of exotropia. In: Burian HM, Dunlap EA, Dyer JA, Fletcher MC, Jampolsky A, Knapp P, et al. editors. Symposium on strabismus: transactions of the New Orleans Academy of Ophthalmology. St Louis: CV Mosby; 1971. p. 233-41.

29. Burian HM, Spivey BE. The surgical management of exodeviations. Trans Am Ophthalmol Soc. 1964;62:276-306. 